PROCEEDINGS OF THE

AMERICAN MATHEMATICAL SOCIETY

Volume 139, Number 10, October 2011, Pages 3727-3734

S 0002-9939(2011)11013-X

Article electronically published on March 30, 2011

\title{
ROOTS OF EHRHART POLYNOMIALS OF GORENSTEIN FANO POLYTOPES
}

\author{
TAKAYUKI HIBI, AKIHIRO HIGASHITANI, AND HIDEFUMI OHSUGI
}

(Communicated by Jim Haglund)

\begin{abstract}
Given arbitrary integers $k$ and $d$ with $0 \leq 2 k \leq d$, we construct a Gorenstein Fano polytope $\mathcal{P} \subset \mathbb{R}^{d}$ of dimension $d$ such that (i) its Ehrhart polynomial $i(\mathcal{P}, n)$ possesses $d$ distinct roots; (ii) $i(\mathcal{P}, n)$ possesses exactly $2 k$ non-real roots and $d-2 k$ real roots; (iii) the real part of each of the non-real roots is equal to $-1 / 2$; (iv) all of the real roots belong to the open interval $(-1,0)$.
\end{abstract}

Recently, many research papers on convex polytopes, including [2], 3], 4], [5], [8], 9] and [18, discuss roots of Ehrhart polynomials. One of the fascinating topics is the study on roots of Ehrhart polynomials of Gorenstein Fano polytopes.

Let $\mathcal{P} \subset \mathbb{R}^{N}$ be an integral convex polytope of dimension $d$ and $\partial \mathcal{P}$ its boundary. (An integral convex polytope is a convex polytope all of whose vertices have integer coordinates.) Given integers $n=1,2, \ldots$, we write $i(\mathcal{P}, n)$ for the number of integer points belonging to $n \mathcal{P}$, where $n \mathcal{P}=\{n \alpha: \alpha \in \mathcal{P}\}$. In other words,

$$
i(\mathcal{P}, n)=\left|n \mathcal{P} \cap \mathbb{Z}^{N}\right|, \quad n=1,2, \ldots
$$

In the late 1950's Ehrhart succeeded in proving that $i(\mathcal{P}, n)$ is a polynomial in $n$ of degree $d$ with $i(\mathcal{P}, 0)=1$. We call $i(\mathcal{P}, n)$ the Ehrhart polynomial of $\mathcal{P}$. Ehrhart's "loi de réciprocité" guarantees that

$$
(-1)^{d} i(\mathcal{P},-n)=i^{*}(\mathcal{P}, n), \quad n=1,2, \ldots,
$$

where $i^{*}(\mathcal{P}, n)=\left|n(\mathcal{P} \backslash \partial \mathcal{P}) \cap \mathbb{Z}^{N}\right|$.

We define the sequence $\delta_{0}, \delta_{1}, \delta_{2}, \ldots$ of integers by the formula

$$
(1-\lambda)^{d+1}\left[1+\sum_{n=1}^{\infty} i(\mathcal{P}, n) \lambda^{n}\right]=\sum_{j=0}^{\infty} \delta_{j} \lambda^{j} .
$$

Since $i(\mathcal{P}, n)$ is a polynomial in $n$ of degree $d$ with $i(\mathcal{P}, 0)=1$, a fundamental fact on generating functions ([21, Corollary 4.3.1]) guarantees that $\delta_{j}=0$ for every $j>d$. The sequence

$$
\delta(\mathcal{P})=\left(\delta_{0}, \delta_{1}, \ldots, \delta_{d}\right)
$$

is called the $\delta$-vector of $\mathcal{P}$. Thus $\delta_{0}=1$ and $\delta_{1}=\left|\mathcal{P} \cap \mathbb{Z}^{N}\right|-(d+1)$. Each $\delta_{j}$ is non-negative (Stanley [20]). If $\delta_{d} \neq 0$, then $\delta_{1} \leq \delta_{j}$ for every $1 \leq j<d([12]$ ).

Received by the editors September 3, 2010.

2010 Mathematics Subject Classification. Primary 52B20; Secondary 52B12.

Key words and phrases. Ehrhart polynomial, $\delta$-vector, Gorenstein Fano polytope.

This research was supported by JST, CREST.

(C)2011 American Mathematical Society Reverts to public domain 28 years from publication 
It follows from loi de réciprocité that

$$
\sum_{n=1}^{\infty} i^{*}(\mathcal{P}, n) \lambda^{n}=\frac{\sum_{j=0}^{d} \delta_{d-j} \lambda^{j+1}}{(1-\lambda)^{d+1}} .
$$

In particular, $\delta_{d}=\left|(\mathcal{P} \backslash \partial \mathcal{P}) \cap \mathbb{Z}^{N}\right|$. Moreover, it follows from (1) that

$$
\max \left\{i: \delta_{i} \neq 0\right\}+\min \left\{i: i(\mathcal{P} \backslash \partial \mathcal{P}) \cap \mathbb{Z}^{N} \neq \emptyset\right\}=d+1 .
$$

We refer the reader to [6], [10, 21], 22], [23] and [24] for further information on Ehrhart polynomials and $\delta$-vectors.

A Fano polytope is an integral convex polytope $\mathcal{P} \subset \mathbb{R}^{d}$ of dimension $d$ such that the origin of $\mathbb{R}^{d}$ is a unique integer point belonging to the interior $\mathcal{P} \backslash \partial \mathcal{P}$ of $\mathcal{P}$. A Fano polytope is called Gorenstein if its dual polytope is integral. (Recall that the dual polytope $\mathcal{P}^{\vee}$ of a Fano polytope $\mathcal{P}$ is the convex polytope which consists of those $x \in \mathbb{R}^{d}$ such that $\langle x, y\rangle \leq 1$ for all $y \in \mathcal{P}$, where $\langle x, y\rangle$ is the usual inner product of $\mathbb{R}^{d}$.) A Gorenstein Fano polytope is often said to be a reflexive polytope. Gorenstein Fano polytopes are classified when $d \leq 4$ in [14 and [15, and the relevance of Gorenstein Fano polytopes to Mirror Symmetry is studied in [1]. We refer the reader to [1], [13], [14, [15] and [17] for related works on toric Fano varieties or Gorenstein toric Fano varieties.

Let $\mathcal{P} \subset \mathbb{R}^{d}$ be a Fano polytope with $\delta(\mathcal{P})=\left(\delta_{0}, \delta_{1}, \ldots, \delta_{d}\right)$ its $\delta$-vector. It follows from [1] and [11] that the following conditions are equivalent:

- $\mathcal{P}$ is Gorenstein;

- $\delta(\mathcal{P})$ is symmetric, i.e., $\delta_{j}=\delta_{d-j}$ for every $0 \leq j \leq d$;

- $i(\mathcal{P}, n)=(-1)^{d} i(\mathcal{P},-n-1)$.

Let $\mathcal{P} \subset \mathbb{R}^{N}$ be an integral convex polytope of dimension $d$ and $i(\mathcal{P}, n)$ its Ehrhart polynomial. A complex number $a \in \mathbb{C}$ is called a root of $i(\mathcal{P}, n)$ if $i(\mathcal{P}, a)=$ 0 . Let $\Re(a)$ denote the real part of $a \in \mathbb{C}$. An outstanding conjecture given in [2] says that every root $a \in \mathbb{C}$ of $i(\mathcal{P}, n)$ satisfies $-d \leq \Re(a) \leq d-1$.

When $\mathcal{P} \subset \mathbb{R}^{d}$ is a Gorenstein Fano polytope, since $i(\mathcal{P}, n)=(-1)^{d} i(\mathcal{P},-n-1)$, the roots of $i(\mathcal{P}, n)$ are distributed symmetrically in the complex plane with respect to the line $\Re(z)=-1 / 2$. Thus, in particular, if $d$ is odd, then $-1 / 2$ is a root of $i(\mathcal{P}, n)$.

It is well-known that the regular unit crosspolytope is a Gorenstein Fano polytope and the $d$ roots of its Ehrhart polynomial have real part $-1 / 2$ for any dimension $d$. It is also known [3, Proposition 1.8] that if all roots $a \in \mathbb{C}$ of $i(\mathcal{P}, n)$ of an integral convex polytope $\mathcal{P} \subset \mathbb{R}^{d}$ of dimension $d$ satisfy $\Re(a)=-1 / 2$, then $\mathcal{P}$ is unimodular isomorphic to a Gorenstein Fano polytope whose volume is at most $2^{d}$. In a recent work [8], the roots of the Ehrhart polynomials of smooth Fano polytopes with small dimensions are completely determined.

In [7, there is a question whether there is a Fano variety the roots $\alpha$ of whose Hilbert polynomial do not satisfy $-1<\Re(\alpha)<0$. Moreover, the vertical line $\Re(\alpha)=-1 / 2$ is the bisector of the vertical strip $-1<\Re(\alpha)<0$. By taking these into consideration, we prove the following:

Theorem 0.1. Given arbitrary non-negative integers $k$ and $d$ with $0 \leq 2 k \leq d$, there exists a Gorenstein Fano polytope $\mathcal{P} \subset \mathbb{R}^{d}$ of dimension d such that

(i) $i(\mathcal{P}, n)$ possesses $d$ distinct roots;

(ii) $i(\mathcal{P}, n)$ possesses exactly $2 k$ non-real roots and $d-2 k$ real roots; 
(iii) the real part of each of the non-real roots is equal to $-1 / 2$;

(iv) all of the real roots belong to the open interval $(-1,0)$.

Proof. Let $\mathbf{e}_{1}, \ldots, \mathbf{e}_{d}$ denote the canonical unit vectors of $\mathbb{R}^{d}$. Let $\mathcal{Q} \subset \mathbb{R}^{d}$ be the convex polytope that is the convex hull of $\mathbf{e}_{1}, \ldots, \mathbf{e}_{2 k}$ and $-\left(\mathbf{e}_{1}+\cdots+\mathbf{e}_{2 k}\right)$. Then $\mathcal{Q}$ is an integral convex polytope of dimension $2 k$ with $\delta(\mathcal{Q})=(1,1, \ldots, 1) \in \mathbb{Z}^{2 k+1}$.

In general, when $\mathcal{F} \subset \mathbb{R}^{N}$ is a $d$-dimensional integral convex polytope, if we define $\mathcal{F}^{\prime} \subset \mathbb{R}^{N+1}$ by setting the convex hull of $\mathcal{F} \cup\left\{\mathbf{e}_{N+1}\right\}$, then one has

$$
i\left(\mathcal{F}^{\prime}, n\right)=1+\sum_{k=1}^{n} i(\mathcal{F}, k) .
$$

It then follows that

$$
\delta\left(\mathcal{F}^{\prime}\right)=(\delta(\mathcal{F}), 0) \in \mathbb{Z}^{d+2} .
$$

Let $\mathcal{Q}^{c} \subset \mathbb{R}^{d}$ be the convex polytope that is the convex hull of $\mathcal{Q} \cup\left\{\mathbf{e}_{2 k+1}, \ldots, \mathbf{e}_{d}\right\}$. Then $\delta\left(\mathcal{Q}^{c}\right)=(\delta(\mathcal{Q}), 0, \ldots, 0) \in \mathbb{Z}^{d+1}$. Hence, by (2), the convex polytope $(d-$ $2 k+1) \mathcal{Q}^{c}$ possesses a unique integer point a in its interior. Now, write $\mathcal{P} \subset \mathbb{R}^{d}$ for the integral convex polytope $(d-2 k+1) \mathcal{Q}^{c}-\mathbf{a}$. Then $\mathcal{P}$ is a Fano polytope.

Since

$$
\sum_{n=0}^{\infty} i\left(\mathcal{Q}^{c}, n\right) \lambda^{n}=\frac{1+\lambda+\lambda^{2}+\cdots+\lambda^{2 k}}{(1-\lambda)^{d+1}}
$$

one has

$$
\begin{aligned}
i\left(\mathcal{Q}^{c}, n\right) & =\sum_{i=n-2 k}^{n}\left(\begin{array}{c}
d+i \\
d
\end{array}\right)=\sum_{i=0}^{2 k}\left(\begin{array}{c}
d+(n-2 k)+i \\
d
\end{array}\right) \\
& =\sum_{i=0}^{2 k}\left(\begin{array}{c}
d+n-(2 k-i) \\
d
\end{array}\right)=\sum_{i=0}^{2 k}\left(\begin{array}{c}
n+d-i \\
d
\end{array}\right) \\
& =\sum_{i=0}^{2 k}\left(\left(\begin{array}{c}
n+d-i+1 \\
d+1
\end{array}\right)-\left(\begin{array}{c}
n+d-i \\
d+1
\end{array}\right)\right) \\
& =\left(\begin{array}{c}
n+d+1 \\
d+1
\end{array}\right)-\left(\begin{array}{c}
n+d-2 k \\
d+1
\end{array}\right) \\
& =\frac{1}{(d+1) !} \prod_{i=1}^{d-2 k}(n+i)\left(\prod_{i=0}^{2 k}(n+d+1-i)-\prod_{i=0}^{2 k}(n-i)\right) .
\end{aligned}
$$

Since

$$
i(\mathcal{P}, n)=i\left((d-2 k+1) \mathcal{Q}^{c}, n\right)=i\left(\mathcal{Q}^{c},(d-2 k+1) n\right),
$$

one has

$$
i(\mathcal{P}, n)=\frac{(d-2 k+1)^{d+1}}{(d+1) !} \prod_{i=1}^{d-2 k}\left(n+\frac{i}{d-2 k+1}\right) F(n)
$$

where

$$
\begin{aligned}
F(n) & =\prod_{i=0}^{2 k}\left(n+\frac{d+1-i}{d-2 k+1}\right)-\prod_{i=0}^{2 k}\left(n-\frac{i}{d-2 k+1}\right) \\
& =\prod_{i=0}^{2 k}\left(n+\frac{d+1-(2 k-i)}{d-2 k+1}\right)-\prod_{i=0}^{2 k}\left(n-\frac{i}{d-2 k+1}\right)
\end{aligned}
$$


Thus we obtain the following equalities:

$$
\begin{aligned}
\prod_{i=1}^{d-2 k}\left(-n-1+\frac{i}{d-2 k+1}\right)=(-1)^{d-2 k} \prod_{i=1}^{d-2 k}\left(n+\frac{d-2 k+1-i}{d-2 k+1}\right) & =(-1)^{d-2 k} \prod_{i=1}^{d-2 k}\left(n+\frac{i}{d-2 k+1}\right) ; \\
F(-n-1)= & \prod_{i=0}^{2 k}\left(-n-1+\frac{d+1-i}{d-2 k+1}\right)-\prod_{i=0}^{2 k}\left(-n-1-\frac{i}{d-2 k+1}\right) \\
= & (-1)^{2 k+1} \prod_{i=0}^{2 k}\left(n+\frac{d-2 k+1-d-1+i}{d-2 k+1}\right) \\
= & (-1)^{2 k} \prod_{i=0}^{2 k}\left(n+\frac{d-2 k+1+i}{d-2 k+1}\right)-(-1)^{2 k} \prod_{i=0}^{2 k}\left(n-\frac{2 k-i}{d-2 k+1}\right) \\
= & (-1)^{2 k} \prod_{i=0}^{2 k}\left(n+\frac{d+1-i}{d-2 k+1}\right)-(-1)^{2 k} \prod_{i=0}^{2 k}\left(n-\frac{d}{d-2 k+1}\right) \\
= & (-1)^{2 k} F(n) .
\end{aligned}
$$

It then follows that

$$
(-1)^{d} i(\mathcal{P},-n-1)=i(\mathcal{P}, n),
$$

which implies that $\mathcal{P}$ is Gorenstein. Hence our work is to show that $\mathcal{P}$ enjoys the required properties (i)-(iv).

Now, since

$$
-\frac{d+1-(2 k-i)}{d-2 k+1}<-\frac{1}{2}<\frac{i}{d-2 k+1}
$$

and since

$$
-\frac{d+1-(2 k-i)}{d-2 k+1}+\frac{i}{d-2 k+1}=-1
$$

Lemma 0.2 below guarantees that $F(n)$ possesses $2 k$ distinct roots, and each of them is a non-real root with $-1 / 2$ its real part. Finally, the real roots of $i(\mathcal{P}, n)$ are

$$
-\frac{i}{d-2 k+1}, \quad 1 \leq i \leq d-2 k
$$

Each of those roots belongs to the open interval $(-1,0)$, as desired.

Lemma 0.2. Let $\alpha_{0}, \alpha_{1}, \ldots, \alpha_{2 k}$ and $\beta_{0}, \beta_{1}, \ldots, \beta_{2 k}$ be rational numbers satisfying $\alpha_{i}<-1 / 2<\beta_{i}$ and $\alpha_{i}+\beta_{i}=-1$ for all $i$. Let

$$
f(x)=\prod_{i=0}^{2 k}\left(x-\alpha_{i}\right)-\prod_{i=0}^{2 k}\left(x-\beta_{i}\right)
$$

be a polynomial in $x$ of degree $2 k$. Then $f(x)$ possesses $2 k$ distinct roots, and each of them is a non-real root with $-1 / 2$ its real part. 
Proof. We employ a basis technique appearing in [19. Let $a \in \mathbb{C}$ with $\Re(a)>-1 / 2$. Since $\alpha_{i}<\beta_{i}$ and $\alpha_{i}+\beta_{i}=-1$, it follows that

$$
\begin{aligned}
\left|a-\alpha_{i}\right|^{2}-\left|a-\beta_{i}\right|^{2} & =\left(\Re(a)-\alpha_{i}\right)^{2}-\left(\Re(a)-\beta_{i}\right)^{2} \\
& =\left(2 \Re(a)-\alpha_{i}-\beta_{i}\right)\left(\beta_{i}-\alpha_{i}\right) \\
& =(2 \Re(a)+1)\left(\beta_{i}-\alpha_{i}\right) \\
& >0 .
\end{aligned}
$$

Hence we have $\left|a-\alpha_{i}\right|>\left|a-\beta_{i}\right|$. Thus $\prod_{i=0}^{2 k}\left|a-\alpha_{i}\right|>\prod_{i=0}^{2 k}\left|a-\beta_{i}\right|$. Hence $f(a) \neq 0$. Similarly, if $a \in \mathbb{C}$ with $\Re(a)<-1 / 2$, then $\left|a-\alpha_{i}\right|<\left|a-\beta_{i}\right|$ for all $i$. Thus $\prod_{i=0}^{2 k}\left|a-\alpha_{i}\right|<\prod_{i=0}^{2 k}\left|a-\beta_{i}\right|$. Hence $f(a) \neq 0$. Consequently, all roots $a \in \mathbb{C}$ of $f(x)$ satisfy $\Re(a)=-1 / 2$.

Substituting $y=x+1 / 2$ and $\gamma_{i}=\beta_{i}+1 / 2$ in $f(x)$, it follows that each of the roots $a \in \mathbb{C}$ of the polynomial

$$
g(y)=\prod_{i=0}^{2 k}\left(\gamma_{i}+y\right)+\prod_{i=0}^{2 k}\left(\gamma_{i}-y\right)
$$

in $y$ of degree $2 k$ satisfies $\Re(a)=0$. Since $\gamma_{i}>0$, one has $g(0) \neq 0$. Hence $g(y)$ possesses no real root. Thus all roots of $f(x)$ are non-real roots.

What we must prove is that $g(y)$ possesses $2 k$ distinct roots. Let $b \in \mathbb{R}$ and let $\theta_{i}(b)$ be the argument of $\gamma_{i}+b \sqrt{-1}$, where $-\pi / 2<\theta_{i}(b)<\pi / 2$. Then $b \sqrt{-1}$ is a root of $g(y)$ if and only if

$$
\prod_{i=0}^{2 k} e^{\sqrt{-1} \theta_{i}(b)}=-\prod_{i=0}^{2 k} e^{-\sqrt{-1} \theta_{i}(b)} .
$$

In other words, $b \sqrt{-1}$ is a root of $g(y)$ if and only if

$$
\prod_{i=0}^{2 k} e^{2 \sqrt{-1} \theta_{i}(b)}=-1,
$$

which is equivalent to saying that

$$
\sum_{i=0}^{2 k} \theta_{i}(b) \equiv \frac{\pi}{2} \quad(\bmod \pi) .
$$

Now, we study the function $h(y)=\sum_{i=0}^{2 k} \theta_{i}(y)$ with $y \in \mathbb{R}$. Since $\gamma_{i}>0$, it follows that $h(y)$ is strictly increasing with

$$
\lim _{y \rightarrow \infty} h(y)=k \pi+\pi / 2, \quad \lim _{y \rightarrow-\infty} h(y)=-(k+1) \pi+\pi / 2 .
$$

Hence the equation

$$
h(y) \equiv \frac{\pi}{2} \quad(\bmod \pi)
$$

possesses $2 k$ distinct real roots, as desired.

Here is an example of Theorem 0.1 .

Example 0.3. Let $k=1$ and $d=4$. Then there exists a 4-dimensional Gorenstein Fano polytope $\mathcal{P} \subset \mathbb{R}^{4}$ such that $i(\mathcal{P}, n)$ satisfies the properties (i)-(iv) of Theorem 0.1 . In fact, we define $\mathcal{Q}^{c}$ by setting the convex hull of

$$
\{(1,0,0,0),(0,1,0,0),(-1,-1,0,0),(0,0,1,0),(0,0,0,1)\} \text {. }
$$


Then $3 \mathcal{Q}^{c}$ contains a unique integer point $(0,0,1,1)$ in its interior. Thus $\mathcal{P}:=$ $3 \mathcal{Q}^{c}-(0,0,1,1)$ is a Gorenstein Fano polytope, which is the convex hull of

$$
\{(3,0,-1,-1),(0,3,-1,-1),(-3,-3,-1,-1),(0,0,2,-1),(0,0,-1,2)\} \text {. }
$$

It can be computed easily that the Ehrhart polynomial of $\mathcal{P}$ is equal to

$$
\frac{81}{8} n^{4}+\frac{81}{4} n^{3}+\frac{135}{8} n^{2}+\frac{27}{4} n+1
$$

and its roots are

$$
-\frac{1}{3},-\frac{2}{3},-\frac{1}{2}+\frac{\sqrt{-7}}{6} \text { and }-\frac{1}{2}-\frac{\sqrt{-7}}{6} .
$$

Remark 0.4. (a) It is disproved in 8 that all of the roots $\alpha$ of the Hilbert polynomial of any Fano variety satisfy $-1<\Re(\alpha)<0$, the so-called canonical strip hypothesis, which is stated in [7. On Theorem 0.1] however, all of the roots of Ehrhart polynomials of our Gorenstein Fano polytopes satisfy this condition. In more detail, they satisfy the narrowed canonical strip hypothesis, which is the condition $-1+1 /(d+1) \leq \Re(\alpha) \leq-1 /(d+1)$. Moreover, if we set $2 k=d$ when $d$ is even or $2 k=d-1$ when $d$ is odd, then they also satisfy the canonical line hypothesis, which is the condition $\Re(\alpha)=-1 / 2$.

(b) We should consider the connections of the Ehrhart polynomials of our Gorenstein Fano polytopes with $L$-functions. Let $i(\mathcal{P}, s)$ be the Ehrhart polynomial of our Gorenstein Fano polytope $\mathcal{P}$ with $2 k=d$ when $d$ is even or with $2 k=d-1$ when $d$ is odd. Then we set $z(s)=i(\mathcal{P},-s)$. Then the function equation

$$
z(1-s)=(-1)^{d} z(s)
$$

holds and all of its roots $\alpha$ satisfy $\Re(\alpha)=1 / 2$, which is, of course, the Riemann zeta function.

Example 0.5. Let $G$ be a finite connected graph on the vertex set $V(G)=$ $\{1, \ldots, d\}$ with $E(G)$ its edge set. We assume that $G$ possesses no loop and no multiple edge. Let $\mathbf{e}_{1}, \ldots, \mathbf{e}_{d}$ denote the canonical unit vectors of $\mathbb{R}^{d}$. For an edge $e=\{i, j\}$ of $G$ with $i<j$, we define $\rho(e)$ and $\mu(e)$ of $\mathbb{R}^{d}$ by setting $\rho(e)=\mathbf{e}_{i}-\mathbf{e}_{j}$ and $\mu(e)=\mathbf{e}_{j}-\mathbf{e}_{i}$. Write $\mathcal{P}_{G}^{ \pm} \subset \mathbb{R}^{d}$ for the convex polytope which is the convex hull of $\{\rho(e): e \in E(G)\} \cup\{\mu(e): e \in E(G)\}$. Let $\mathcal{H} \subset \mathbb{R}^{d}$ denote the hyperplane defined by the equation $\sum_{i=1}^{d} x_{i}=0$. Then $\mathcal{P}_{G}^{ \pm} \subset \mathcal{H}$. Identifying $\mathcal{H}$ with $\mathbb{R}^{d-1}$, it turns out that $\mathcal{P}_{G}^{ \pm} \subset \mathbb{R}^{d-1}$ is a Gorenstein Fano polytope of dimension $d-1$. (In detail, see [16, Proposition 3.2].) One of the research problems is to find a combinatorial characterization of the finite graphs $G$ for which all roots $a \in \mathbb{C}$ of $i\left(\mathcal{P}_{G}^{ \pm}, n\right)$ satisfy $\Re(a)=-1 / 2$.

For example, if $C$ is a cycle of length 6 , then all roots $a \in \mathbb{C}$ of $i\left(\mathcal{P}_{C}^{ \pm}, n\right)$ satisfy $\Re(a)=-1 / 2$. However, if $C$ is a cycle of length 7 , then there is a root $a \in \mathbb{C}$ of $i\left(\mathcal{P}_{C}^{ \pm}, n\right)$ with $\Re(a) \neq-1 / 2$.

If $G$ is a tree, then $\mathcal{P}_{G}^{ \pm}$is unimodular isomorphic to the regular unit crosspolytope of dimension $d-1$, which is the convex hull of $\left\{ \pm \mathbf{e}_{1}, \ldots, \pm \mathbf{e}_{d-1}\right\}$ in $\mathbb{R}^{d-1}$. Hence the $\delta$-vector of $\mathcal{P}_{G}^{ \pm}$is $\delta\left(\mathcal{P}_{G}^{ \pm}\right)=\left(\left(\begin{array}{c}d-1 \\ 0\end{array}\right),\left(\begin{array}{c}d-1 \\ 1\end{array}\right), \ldots,\left(\begin{array}{l}d-1 \\ d-1\end{array}\right)\right)$. Thus, by using [19] again, all roots $a \in \mathbb{C}$ of $i\left(\mathcal{P}_{G}^{ \pm}, n\right)$ satisfy $\Re(a)=-1 / 2$. 
Let $G$ be a complete bipartite graph of type $(2, d-2)$. Thus the edges of $G$ are either $\{1, j\}$ or $\{2, j\}$ with $3 \leq j \leq d$. Let $\delta\left(\mathcal{P}_{G}^{ \pm}\right)=\left(\delta_{0}, \delta_{1}, \ldots, \delta_{d-1}\right)$. Then

$$
\sum_{k=0}^{d-1} \delta_{k} x^{k}=(1+x)^{d-3}\left(1+2(d-2) x+x^{2}\right) .
$$

It has been conjectured that all roots $a \in \mathbb{C}$ of $i\left(\mathcal{P}_{G}^{ \pm}, n\right)$ satisfy $\Re(a)=-1 / 2$ in [16].

\section{REFERENCES}

[1] V. Batyrev, Dual polyhedra and mirror symmetry for Calabi-Yau hypersurfaces in toric varieties, J. Algebraic Geom. 3 (1994), 493-535. MR1269718 (95c:14046)

[2] M. Beck, J. A. De Loera, M. Develin, J. Pfeifle and R. P. Stanley, Coefficients and roots of Ehrhart polynomials, Contemp. Math. 374, Amer. Math. Soc., 2005, 15-36. MR 2134759 (2006e:52019)

[3] C. Bey, M. Henk and J. M. Wills, Notes on the roots of Ehrhart polynomials, Discrete Comput. Geom. 38 (2007), 81-98. MR2322117(2008k:52027)

[4] B. Braun, Norm bounds for Ehrhart polynomial roots, Discrete Comput. Geom. 39 (2008), 191-193. MR2383758(2009d:52019)

[5] B. Braun and M. Develin, Ehrhart polynomial roots and Stanley's non-negativity theorem, Contemp. Math. 452, Amer. Math. Soc., 2008, 67-78. MR2405764 (2009f:52040)

[6] E. Ehrhart, "Polynômes Arithmétiques et Méthode des Polyèdres en Combinatoire," Birkhäuser, Boston/Basel/Stuttgart, 1977. MR0432556 (55:5544)

[7] V. V. Golyshev, On the canonical strip, Russ. Math. Surv. 64 (2009), no. 1, 145-147. MR2503098 (2010g:14060)

[8] G. Hegedüs and A. M. Kasprzyk, Roots of Ehrhart polynomials of smooth Fano polytopes, Discrete Comput. Geom. (2010).

[9] M. Henk, A. Schürmann and J. M. Wills, Ehrhart polynomials and successive minima, Mathematika 52 (2005), 1-16. MR2261838(2007h:52013)

[10] T. Hibi, "Algebraic Combinatorics on Convex Polytopes," Carslaw Publications, Glebe, N.S.W., Australia, 1992.

[11] T. Hibi, Dual polytopes of rational convex polytopes, Combinatorica 12 (1992), 237-240. MR:1179260 (93f:52018)

[12] T. Hibi, A lower bound theorem for Ehrhart polynomials of convex polytopes, Adv. in Math. 105 (1994), 162-165. MR.1275662 (95b:52018)

[13] A. M. Kasprzyk, Toric Fano threefolds with terminal singularities, Tohoku Math. J. (2) 58 (2006), no. 1, 101-121. MR2221794 (2007b:14089)

[14] M. Kreuzer and H. Skarke, On the classification of reflexive polyhedra, Comm. Math. Phys. 185 (1997), no. 2, 495-508. MR1463052 (98f:32029)

[15] M. Kreuzer and H. Skarke, Complete classification of reflexive polyhedra in four dimensions, Adv. Theor. Math. Phys. 4 (2000), no. 6, 1209-1230. MR1894855(2003e:52020)

[16] T. Matsui, A. Higashitani, Y. Nagazawa, H. Ohsugi and T. Hibi, Roots of Ehrhart polynomials arising from graphs, Journal of Algebraic Combinatorics, to appear.

[17] B. Nill, Gorenstein toric Fano varieties, Manuscripta Math. 116 (2005), no. 2, 183-210. MR2122419 (2005k:14110)

[18] J. Pfeifle, Gale duality bounds for roots of polynomials with nonnegative coefficients, J. Comb. Theory Ser. A 117 (2010), no. 3, 248-271. MR2592900

[19] F. Rodriguez-Villegas, On the zeros of certain polynomials, Proc. Amer. Math. Soc. 130 (2002), 2251-2254. MR1896405(2003d:12002)

[20] R. P. Stanley, Decompositions of rational convex polytopes, Annals of Discrete Math. 6 (1980), 333-342. MR593545 (82a:52007)

[21] R. P. Stanley, "Enumerative Combinatorics, Volume 1," Wadsworth \& Brooks/Cole, Monterey, Calif., 1986. MR 0847717 (87j:05003)

[22] R. P. Stanley, On the Hilbert function of a graded Cohen-Macaulay domain, J. Pure and Appl. Algebra 73 (1991), 307-314. MR1124790 (92f:13017) 
[23] R. P. Stanley, A monotonicity property of $h$-vectors and $h^{*}$-vectors, Europ. J. Combinatorics 14 (1993), 251-258. MR1215335 (94f:52016)

[24] R. P. Stanley, "Combinatorics and Commutative Algebra," Second Ed., Birkhäuser, 1996. MR:1453579 (98h:05001)

Department of Pure and Applied Mathematics, Graduate School of Information SciEnce And Technology, Osaka University, Toyonaka, Osaka 560-0043, Japan

E-mail address: hibi@math.sci.osaka-u.ac.jp

Department of Pure and Applied Mathematics, Graduate School of Information Science and Technology, Osaka University, Toyonaka, Osaka 560-0043, Japan

E-mail address: sm5037ha@ecs.cmc.osaka-u.ac.jp

Department of Mathematics, College of Science, Rikkyo University, Toshima-ku, TOKYO 171-8501, JAPAN

E-mail address: ohsugi@rikkyo.ac.jp 